\title{
Enhanced Catechol Oxidase and Peroxidase Activities as Possible Markers for Measuring Chromium Tolerance in Sorghum (Sorghum bicolor L.) Genotypes
}

\author{
Hajo Elzein Elhassan ${ }^{1 *}$, Abdel Wahab Hassan Abdalla ${ }^{2}$, El Busra El Shiekh. El Nur ${ }^{3}$ \\ ${ }^{1}$ Environment, Natural Resources and Desertification Research Institute, National Centre for Research P. O. Box \\ 6096 Khartoum Sudan \\ ${ }^{2}$ Department of Agronomy, Faculty of Agriculture, University of Khartoum, Khartoum, Sudan \\ ${ }^{3}$ Department of Botany, Faculty of Science, University of Khartoum, Khartoum, Sudan
}

Received: 24 May 2015 / Accepted: 20 September 2015

*Corresponding author: elhassanhajo@yahoo.com

\begin{abstract}
The aim of the present study was to estimate the effect of chromium on catechol oxidase and peroxidase activities in ten sorghum genotypes grown in soil amended by different concentrations of chromium. Ten sorghum genotypes, (Tabat, Wad Ahmed, L4, L7, L12, L14, L16, L25, L32 and L34) were obtained from the Department of Agronomy, Faculty of Agriculture University of Khartoum., Seeds were sown in polyethylene bags filled with $2 \mathrm{~kg}$ soil (clay and sand, 2:1). Two weeks after sowing, the seedlings were irrigated with eight levels of chromium (Cr-VI) concentrations $(0,2.5,5,10,20,30,40$, and $50 \mathrm{mg} / \mathrm{l}$.). Samples for assaying enzyme (catechol oxidase and peroxidase) activities were taken three times (on the 8, 12, and 15 day) following chromium application. The results revealed that the level of enzyme activity of most genotypes was increased significantly with increasing chromium concentration. The magnitude of increase in enzymatic activity was also dependent on the genotype and duration of time after chromium treatment. These findings suggest that, the activities of both enzymes might be used as indicator for selecting chromium tolerant sorghum genotypes.
\end{abstract}

Keywords: chromium, catechol oxidase, peroxidase, sorghum, genotypes.

\section{Introduction}

Chromium is important for metallurgical industry. Chromium salts are used in many industrial processes and product such as, steel production, electroplating, leather tanning, metal finishing, inhibition of metal corrosion, textile paints and pigment manufacture, catalysts applications, drilling muds, fungicides and nuclear weapons production. Therefore, chromium salts are frequently present in effluents of those industries and in municipal sewage (Wong et al., 2001; Zayed and Terry 2003; Nath et al., 2005; Babel and Opiso, 2007; Venkateswaran et al., 2007) 
Chromium (Cr) occurs in nature in bound forms that constitute $0.1-0.3 \mathrm{mg} \mathrm{kg}^{-1}$ of the Earth's crust and has several oxidation states from $\mathrm{Cr}(-\mathrm{II})$ to $\mathrm{Cr}(+\mathrm{VI})$ (Zayed and Terry, 2003). It is unique among the heavy metals because of its existence in two environmentally important oxidation states: trivalent (Cr III) and hexavalent ( $\mathrm{Cr} \quad \mathrm{VI})$ (Srivastava et al., 1999). Chromium is recognized as an essential element for humans and animals (Mertz, 1967), but not for plants (Huffman and Allaway, 1973; Liu et al., 1992), although some investigations report that it is beneficial to plant growth (Zheng et al., 1987).

Symptoms of Cr phytotoxicity include inhibition of seed germination or of early seedling development, reduction of root growth, leaf chlorosis and depressed biomass (Sharma et al., 1995). Chromium significantly affects the metabolism of plants such as barley (Hordeumvulgare) (Ali et al., 2004), citrullus (Dube et al., 2003), cauliflower (Chatterjee and Chatterjee, 2000), vegetable crops (Zayed et al., 1998), wheat (Triticum aestivumcv. HD2204) (Sharma et al., 1995) and maize (Zea mays) (Sharma and Pant, 1994). Chromium toxicity in plants also leads to leaves chlorosis, tissue necrosis, decreases enzyme activity, causes membrane damage, diminished photosynthesis and changing of chloroplast (Jain et al., 2000; Parmar et al., 2002; Du etal., 2003; Dube et al., 2003; Zayed and Terry 2003; Scoccianti et al., 2006). Therefore the aim of the present study was to investigate the effects of different concentrations of chromium on catechol oxidase and proxidase activity in ten sorghum genotypes seedling.

\section{Materials and Methods}

Seeds of ten sorghum genotypes,(Tabat, Wad Ahmed, L4, L7, L12, L14, L16, L25, L32 and L34) were obtained from the Department of Agronomy, Faculty of Agriculture, University of Khartoum. Seeds were germinated, on the third week of July, in polyethylene bags; each filled with $2 \mathrm{~kg}$ soil consisted of a mixture of clay and sand (2: 1). Eight chromium concentrations $(0$, $2.5,5,10,20,30,40$, and $50 \mathrm{mg} \mathrm{l}^{-1}$.) were applied, using potassium dichromate as a source of chromium. Six seeds per pot were sown on the third week of July 2011 and routinely irrigated with tap water. Growth conditions two weeks after sowing, the plants were irrigated with the differences concentrations of chromium. The data were taken on $8^{\text {th, }} 12^{\text {th }}$, and $15^{\text {th }}$ day after chromium application to measure the activities of catechol oxidase and peroxidase enzymes. Enzymes were extracted from plant leaves (second fully expanded leaf from the top) at harvest

\section{Enzymes extraction:}

Three $\mathrm{g}$ of fresh leaves of plants from each genotype were homogenized with $100 \mathrm{mM}$ potassium phosphate buffer ( $\mathrm{pH} 7.5)$ and $1 \mathrm{mM}$ EDTA. The homogenate tissue was filtered through four layers of cheesecloth and then centrifuged at $13,000 \mathrm{rpm}$ at $4^{\circ} \mathrm{C}$ for 10 minutes, using a Sigma Laboratory Refrigerated Centrifuge. The supernatant was used for the enzyme assays. Protein content was determined using bovine serum albumin as standard Bradford (1976).

\section{Catechol oxidase (1.10.3.1):}

Catechol oxidase activity was determined using substrate concentration as $3 \mathrm{mM}$ catechol in 50 $\mathrm{mM}$ potassium phosphate buffer $(\mathrm{pH} 6.5)$ at $25^{\circ} \mathrm{C}$ by monitoring the increase of absorbance at 420 $\mathrm{nm}$. Enzyme specific activity is expressed as $\mathrm{U}$ per mg protein (Tremoliere and Bieth1984)

\section{Peroxidase (1.11.1.7):}

Peroxidase activity was measured [19]. The reaction mixture contained $50 \mathrm{mM}$ potassium phosphate buffer (pH 7.0), 0.1 mM EDTA, $40 \mathrm{mM}$ $\mathrm{H}_{2} \mathrm{O}_{2}$, and $0.5 \mathrm{mM}$ pyrogallol and $0.5 \mathrm{ml}$ enzymes with final volume $3 \mathrm{ml}$. Absorbance was read at $430 \mathrm{~nm}$. Enzyme activity was expressed as $\mu \mathrm{mol}$ $\mathrm{H}_{2} \mathrm{O}_{2}$ destroyed $\mathrm{min}^{-1} \mathrm{mg}^{-1}$ protein (Mandelman $e t$ al. 1998).

\section{Statistical analysis:}

Data were analyzed as complete randomized design with two factors. Analysis of variance (ANOVA) was performed. Means were separated using Duncan Multiple Range Test (Gomez and Gomez1984).

\section{Results}

The effect of chromium on catechol oxidase activity of ten sorghum genotypes on the $8^{\text {th, }} 12^{\text {th }}$ and $15^{\text {th }}$ of treatment is shown in Tables 1,2 and 3, respectively. In relation to corresponding controls, 
catechol oxidase activity significantly increased with increasing chromium concentration. The (50 $\mathrm{mgl}^{-1}$ ) of chromium elevated the enzyme activity by a range of 355 in L14 to $1115 \%$ in L4 on the $8^{\text {th }}$ of treatment; however, there was a decrease in L25, L32 and L34. Similarly, catechol oxidase activity was increased by $50 \mathrm{ppm}$ of chromium on the $12^{\text {th }}$ day by a range of 163 in L12 to $337 \%$ in L32; however, a decrease was detected in L7. Also, $50 \mathrm{ppm}$ of chromium increased catechol oxidase activity on the $15^{\text {th }}$ day bly a range of 149 $\%$ in L25 to $2338 \%$ in L34

Table 4 represents the effect of chromium on peroxidase activity of ten sorghum genotypes on the $8^{\text {th }}$ of treatment. As compared to control, peroxidase activity significantly increased with increasing chromium concentration throughout the entire period in spite of the decreases detected of the enzyme activity in L25, L32 and L34 genotypes. Similar increases were also detected on the $12^{\text {th }}$ and $15^{\text {th }}$ of treatment (Tables 5 and 6 , respectively). The highest concentration (50 ppm) of chromium induced an increase of about $211 \%$ in Wad Ahmed to $1200 \%$ in L14 on the $8^{\text {th }}$ of treatment with a decrease in L25, L32 and L34. Peroxidase activity was increased by $50 \mathrm{ppm}$ of chromium on the $12^{\text {th }}$ day by a range of 103 in L7 to $246 \%$ in L16. Also, $50 \mathrm{ppm}$ of chromium increased peroxidase activity on the $15^{\text {th }}$ day by a range of $217 \%$ in L25 to1800\% in Tabat.

Table 1 Effect of chromium on catechol oxidase activity (u/mg protein) of the ten sorghum genotypes, after 8 days of chromium application (at eight different concentrations) to two-week-old seedlings.

\begin{tabular}{|c|c|c|c|c|c|c|c|c|c|c|c|}
\hline \multirow[b]{2}{*}{$\begin{array}{c}\text { Chromium } \\
\text { concentratio } \\
\text { ns (ppm) }\end{array}$} & \multicolumn{10}{|c|}{ Sorghum genotypes } & \multirow[b]{2}{*}{$\begin{array}{c}\text { Chromium } \\
\text { means }\end{array}$} \\
\hline & Tabat & $\begin{array}{c}\text { Wad } \\
\text { aAhme } \\
\text { d }\end{array}$ & L4 & L7 & L12 & L14 & L16 & L25 & L32 & L34 & \\
\hline 0 & $0.65^{\mathrm{i}}$ & $1.83^{\text {cdefgi }}$ & $0.64^{\mathrm{i}}$ & $0.68^{\text {hi }}$ & $0.99^{\text {ghi }}$ & $0.61^{\mathrm{i}}$ & $0.50^{\mathrm{i}}$ & $\begin{array}{c}3.13^{\text {bcdefgh }} \\
\mathrm{i}\end{array}$ & $\begin{array}{c}3.11_{\mathrm{i}}^{\text {bcdefgh }} \\
\text { be }\end{array}$ & $1.92^{\text {cdefghi }}$ & $1.41^{\mathrm{c}}$ \\
\hline 2.5 & $1.50^{\text {defghi }}$ & $1.88^{\text {cdefgi }}$ & $0.79^{\text {hi }}$ & $1.01^{\text {ghi }}$ & $0.68^{\mathrm{hi}}$ & $0.81^{\mathrm{hi}}$ & $0.71^{\mathrm{hi}}$ & $1.46^{\text {defghi }}$ & $3.79^{\text {bcdefg }}$ & $2.23^{\text {cdefghi }}$ & $1.49^{\mathrm{c}}$ \\
\hline 5 & $1.52^{\text {defghi }}$ & $2.24^{\text {cdefghi }}$ & $1.18^{\text {efghi }}$ & $0.89^{\mathrm{hi}}$ & $1.05^{\text {fghi }}$ & $1.56^{\text {defghi }}$ & $0.65^{\mathrm{i}}$ & $1.40^{\mathrm{defghi}}$ & $\begin{array}{c}2.56^{\text {bcdefgh }} \\
i\end{array}$ & $1.70^{\text {cdefghi }}$ & $1.47^{\mathrm{c}}$ \\
\hline 10 & $2.19^{\text {cdefghi }}$ & $2.81^{\text {bcdefghi }}$ & $1.85^{\text {cdefghi }}$ & $1.44^{\text {defgh }}$ & $1.78^{\text {cdefghi }}$ & $1.23^{\text {efghi }}$ & $1.31^{\text {efghi }}$ & $1.68^{\text {defghi }}$ & $1.17^{\text {efghi }}$ & $1.10^{\text {fghi }}$ & $1.66^{\mathrm{c}}$ \\
\hline 20 & $2.10^{\text {cdefghi }}$ & $2.55^{\text {bcdefghi }}$ & $2.32^{\text {cdefghi }}$ & $2.41^{\text {bcdeefghi }}$ & $2.84_{i}^{\text {bcdefgh }}$ & $1.56^{\text {defghi }}$ & $0.92^{\text {ghi }}$ & $1.23^{\text {efghi }}$ & $0.76^{\mathrm{hi}}$ & $0.90^{\text {ghi }}$ & $1.76^{\mathrm{bc}}$ \\
\hline 30 & $2.15^{\text {cdefghi }}$ & $3.71^{\text {bcdefgh }}$ & $\begin{array}{c}3.03^{\text {bcdefgh }} \\
\mathrm{i}\end{array}$ & $2.36^{\text {cdefghi }}$ & $4.27^{\mathrm{abcd}}$ & $1.91_{\mathrm{i}}^{\text {cdefgh }}$ & $1.65^{\text {defghi }}$ & $1.10^{\text {fghi }}$ & $0.73^{\mathrm{hi}}$ & $0.92^{\text {ghi }}$ & $2.18^{\mathrm{bc}}$ \\
\hline 40 & $4.00^{\text {bcde }}$ & $4.04^{\text {bcde }}$ & $3.63^{\text {bcdefgh }}$ & $3.91^{\text {bcdef }}$ & $4.35^{\text {abcd }}$ & $1.81_{i}^{\text {cdefgh }}$ & $\begin{array}{c}2.08^{\text {cdefgh }} \\
\text { i }\end{array}$ & $0.78^{\text {hi }}$ & $0.66^{\mathrm{hi}}$ & $0.67^{\mathrm{hi}}$ & $2.59^{\mathrm{ab}}$ \\
\hline 50 & $4.64^{\mathrm{abc}}$ & $4.28^{\mathrm{abcd}}$ & $7.14^{\mathrm{a}}$ & $3.76^{\text {bcdefg }}$ & $5.25^{\mathrm{ab}}$ & $\begin{array}{c}2.17^{\text {cdefgh }} \\
i\end{array}$ & $\begin{array}{c}2.35^{\text {cdefgh }} \\
i\end{array}$ & $0.75^{\mathrm{hi}}$ & $0.92^{\text {ghi }}$ & $0.61^{\mathrm{i}}$ & $3.19^{\mathrm{a}}$ \\
\hline $\begin{array}{c}\text { Genotypes } \\
\text { means }\end{array}$ & $2.34^{\mathrm{abc}}$ & $2.92^{\mathrm{a}}$ & $2.57^{\mathrm{ab}}$ & $2.06^{\mathrm{abcd}}$ & $2.65^{\mathrm{ab}}$ & $1.46^{\mathrm{cd}}$ & $1.27^{\mathrm{d}}$ & $1.44^{\mathrm{cd}}$ & $1.71^{\mathrm{bcd}}$ & $1.26^{\mathrm{d}}$ & \\
\hline
\end{tabular}

Potassium dichromate was used as a source of chromium. The soil used was clay: sand (2:1). Statistical analysis was done using Duncan multiple range test (DMRT). Values with the same superscript letters are not significantly different at $\mathrm{p}<0.05$

Table 2 Effect of chromium on catechol oxidase activity ( $\mathrm{u} / \mathrm{mg}$ protein) of the ten sorghum genotypes at the $12^{\text {th }}$ day after chromium application (at eight different concentrations) to two-week-old seedlings

\begin{tabular}{|c|c|c|c|c|c|c|c|c|c|c|c|c|}
\hline $\begin{array}{c}\text { Chromium } \\
\text { concentrations } \\
(\mathbf{p p m})\end{array}$ & Tabat & $\begin{array}{c}\text { Wad } \\
\text { ahmed }\end{array}$ & $\mathbf{L 4}$ & $\mathbf{L 7}$ & $\mathbf{L 1 2}$ & $\mathbf{L 1 4}$ & $\mathbf{L 1 6}$ & $\mathbf{L 2 5}$ & $\mathbf{L 3 2}$ & L34 & $\begin{array}{c}\text { Chromium } \\
\text { means }\end{array}$ \\
\hline & $0.76^{\mathrm{a}}$ & $1.39^{\mathrm{a}}$ & $0.86^{\mathrm{a}}$ & $3.66^{\mathrm{a}}$ & $1.24^{\mathrm{a}}$ & $0.80^{\mathrm{a}}$ & $0.94^{\mathrm{a}}$ & $1.23^{\mathrm{a}}$ & $1.20^{\mathrm{a}}$ & $1.43^{\mathrm{a}}$ & $1.35^{\mathrm{d}}$ \\
\hline 0 & $0.80^{\mathrm{a}}$ & $1.48^{\mathrm{a}}$ & $0.82^{\mathrm{a}}$ & $3.60^{\mathrm{a}}$ & $1.55^{\mathrm{a}}$ & $0.73^{\mathrm{a}}$ & $0.91^{\mathrm{a}}$ & $1.55^{\mathrm{a}}$ & $1.16^{\mathrm{a}}$ & $2.33^{\mathrm{a}}$ & $1.49^{\mathrm{cd}}$ \\
\hline 2.5 & $0.94^{\mathrm{a}}$ & $1.49^{\mathrm{a}}$ & $1.54^{\mathrm{a}}$ & $3.72^{\mathrm{a}}$ & $1.61^{\mathrm{a}}$ & $0.89^{\mathrm{a}}$ & $0.95^{\mathrm{a}}$ & $1.43^{\mathrm{a}}$ & $1.21^{\mathrm{a}}$ & $2.74^{\mathrm{a}}$ & $1.65^{\mathrm{bcd}}$ \\
\hline 5 & $0.94^{\mathrm{a}}$ & $1.59^{\mathrm{a}}$ & $1.58^{\mathrm{a}}$ & $3.03^{\mathrm{a}}$ & $1.59^{\mathrm{a}}$ & $0.95^{\mathrm{a}}$ & $1.14^{\mathrm{a}}$ & $1.57^{\mathrm{a}}$ & $1.61^{\mathrm{a}}$ & $2.51^{\mathrm{a}}$ & $1.65^{\mathrm{bcd}}$ \\
\hline 10 & $0.97^{\mathrm{a}}$ & $1.77^{\mathrm{a}}$ & $1.90^{\mathrm{a}}$ & $2.40^{\mathrm{a}}$ & $1.71^{\mathrm{a}}$ & $1.17^{\mathrm{a}}$ & $1.10^{\mathrm{a}}$ & $1.58^{\mathrm{a}}$ & $1.56^{\mathrm{a}}$ & $2.68^{\mathrm{a}}$ & $1.68^{\mathrm{bcd}}$ \\
\hline 20 & $1.33^{\mathrm{a}}$ & $1.95^{\mathrm{a}}$ & $2.00^{\mathrm{a}}$ & $2.33^{\mathrm{a}}$ & $1.80^{\mathrm{a}}$ & $1.47^{\mathrm{a}}$ & $1.21^{\mathrm{a}}$ & $1.68^{\mathrm{a}}$ & $2.13^{\mathrm{a}}$ & $2.72^{\mathrm{a}}$ & $1.86^{\mathrm{bc}}$ \\
\hline 30 & $1.20^{\mathrm{a}}$ & $2.21^{\mathrm{a}}$ & $2.21^{\mathrm{a}}$ & $2.02^{\mathrm{a}}$ & $1.85^{\mathrm{a}}$ & $1.87^{\mathrm{a}}$ & $1.89^{\mathrm{a}}$ & $2.45^{\mathrm{a}}$ & $2.36^{\mathrm{a}}$ & $2.74^{\mathrm{a}}$ & $2.08^{\mathrm{b}}$ \\
\hline $\begin{array}{c}\text { Genotypes } \\
\text { means }\end{array}$ & $1.49^{\mathrm{a}}$ & $2.25^{\mathrm{a}}$ & $2.37^{\mathrm{a}}$ & $2.17^{\mathrm{a}}$ & $1.83^{\mathrm{a}}$ & $2.14^{\mathrm{a}}$ & $2.60^{\mathrm{a}}$ & $3.62^{\mathrm{a}}$ & $4.04^{\mathrm{a}}$ & $4.26^{\mathrm{a}}$ & $2.68^{\mathrm{a}}$ \\
\hline 50 & $1.06^{\mathrm{e}}$ & $1.77^{\mathrm{bcd}}$ & $1.66^{\mathrm{bcd}}$ & $2.87^{\mathrm{a}}$ & $1.65^{\mathrm{bcd}}$ & $1.25^{\mathrm{de}}$ & $1.34^{\mathrm{cd}}$ & $1.89^{\mathrm{bc}}$ & $1.91^{\mathrm{b}}$ & $2.68^{\mathrm{a}}$ & \\
\hline
\end{tabular}

Potassium dichromate was used as a source of chromium. The soil used was clay: sand (2:1). Statistical analysis was done using Duncan multiple range test (DMRT). Values with the same superscript letters are not significantly different at $\mathrm{p}<0.05$ 
Table 3 Effect of chromium on catechol oxidase activity ( $\mathrm{u} / \mathrm{mg}$ protein) of the ten sorghum genotypes at the $15^{\text {th }}$ day after chromium application at eight different concentrations) to two-week-old seedlings

\begin{tabular}{|c|c|c|c|c|c|c|c|c|c|c|c|}
\hline \multirow{2}{*}{$\begin{array}{c}\text { Chromium } \\
\text { concentrations } \\
(\mathbf{p p m})\end{array}$} & \multicolumn{10}{|c|}{ Sorghum genotypes } & \multirow[b]{2}{*}{$\begin{array}{c}\text { Chromium } \\
\text { means }\end{array}$} \\
\hline & Tabat & $\begin{array}{c}\text { Wad } \\
\text { ahmed }\end{array}$ & L4 & L7 & L12 & L14 & L16 & L25 & L32 & L34 & \\
\hline 0 & $0.11^{\mathrm{a}}$ & $0.45^{\mathrm{a}}$ & $0.40^{\mathrm{a}}$ & $0.28^{\mathrm{a}}$ & $0.56^{\mathrm{a}}$ & $0.42^{\mathrm{a}}$ & $0.44^{\mathrm{a}}$ & $1.56^{\mathrm{a}}$ & $0.99^{\mathrm{a}}$ & $0.13^{\mathrm{a}}$ & $0.53^{\mathrm{e}}$ \\
\hline 2.5 & $0.08^{\mathrm{a}}$ & $0.61^{\mathrm{a}}$ & $0.54^{\mathrm{a}}$ & $0.64^{\mathrm{a}}$ & $0.67^{\mathrm{a}}$ & $0.50^{\mathrm{a}}$ & $0.45^{\mathrm{a}}$ & $1.57^{\mathrm{a}}$ & $0.83^{\mathrm{a}}$ & $1.14^{\mathrm{a}}$ & $0.70^{\mathrm{e}}$ \\
\hline 5 & $0.22^{\mathrm{a}}$ & $0.87^{\mathrm{a}}$ & $0.63^{\mathrm{a}}$ & $1.30^{\mathrm{a}}$ & $0.67^{\mathrm{a}}$ & $0.61^{\mathrm{a}}$ & $0.43^{\mathrm{a}}$ & $1.50^{\mathrm{a}}$ & $1.57^{\mathrm{a}}$ & $1.38^{\mathrm{a}}$ & $0.92^{\mathrm{e}}$ \\
\hline 10 & $0.20^{\mathrm{a}}$ & $1.60^{\mathrm{a}}$ & $0.73^{\mathrm{a}}$ & $1.38^{\mathrm{a}}$ & $0.73^{\mathrm{a}}$ & $1.30^{\mathrm{a}}$ & $0.48^{\mathrm{a}}$ & $2.00^{\mathrm{a}}$ & $1.71^{\mathrm{a}}$ & $1.47^{\mathrm{a}}$ & $1.16^{\mathrm{cd}}$ \\
\hline 20 & $0.08^{\mathrm{a}}$ & $2.60^{\mathrm{a}}$ & $1.10^{\mathrm{a}}$ & $1.44^{\mathrm{a}}$ & $0.77^{\mathrm{a}}$ & $1.26^{\mathrm{a}}$ & $0.69^{\mathrm{a}}$ & $2.09^{\mathrm{a}}$ & $2.04^{\mathrm{a}}$ & $1.96^{\mathrm{a}}$ & $1.40^{\mathrm{bc}}$ \\
\hline 30 & $0.44^{\mathrm{a}}$ & $2.59^{\mathrm{a}}$ & $1.39^{\mathrm{a}}$ & $1.58^{\mathrm{a}}$ & $1.05^{\mathrm{a}}$ & $1.36^{\mathrm{a}}$ & $1.04^{\mathrm{a}}$ & $2.20^{\mathrm{a}}$ & $2.15^{\mathrm{a}}$ & $2.56^{\mathrm{a}}$ & $1.64^{\mathrm{b}}$ \\
\hline 40 & $0.44^{\mathrm{a}}$ & $2.77^{\mathrm{a}}$ & $1.78^{\mathrm{a}}$ & $1.38^{\mathrm{a}}$ & $1.02^{\mathrm{a}}$ & $1.49^{\mathrm{a}}$ & $1.50^{\mathrm{a}}$ & $2.24^{\mathrm{a}}$ & $2.53^{\mathrm{a}}$ & $2.52^{\mathrm{a}}$ & $1.77^{\mathrm{ab}}$ \\
\hline 50 & $0.44^{\mathrm{a}}$ & $3.27^{\mathrm{a}}$ & $2.04^{\mathrm{a}}$ & $1.87^{\mathrm{a}}$ & $1.68^{\mathrm{a}}$ & $2.37^{\mathrm{a}}$ & $1.85^{\mathrm{a}}$ & $2.33^{\mathrm{a}}$ & $2.91^{\mathrm{a}}$ & $3.04^{\mathrm{a}}$ & $2.18^{\mathrm{a}}$ \\
\hline $\begin{array}{c}\text { Genotypes } \\
\text { means }\end{array}$ & $0.25^{\mathrm{c}}$ & $1.84^{\mathrm{a}}$ & $1.08^{\mathrm{b}}$ & $1.24^{\mathrm{b}}$ & $0.89^{\mathrm{b}}$ & $1.16^{\mathrm{b}}$ & $0.86^{\mathrm{b}}$ & $1.94^{\mathrm{a}}$ & $1.84^{\mathrm{a}}$ & $1.78^{\mathrm{a}}$ & \\
\hline
\end{tabular}

Potassium dichromate was used as a source of chromium. The soil used was clay: sand (2:1). Statistical analysis was done using Duncan multiple range test (DMRT). Values with the same superscript letters are not significantly different at $\mathrm{p}<0.05$

Table 4 Effect of chromium on perooxidase activity ( $\mathrm{u} / \mathrm{mg}$ protein) of the ten sorghum genotypes at the $8^{\text {th }}$ day after chromium application at eight different concentrations) to two-week-old seedlings

\begin{tabular}{|c|c|c|c|c|c|c|c|c|c|c|c|}
\hline \multirow{2}{*}{\begin{tabular}{|c|} 
Chromium \\
concentration \\
s (ppm) \\
\end{tabular}} & \multicolumn{10}{|c|}{ Sorghum genotypes } & \multirow{2}{*}{$\begin{array}{l}\text { Chrom } \\
\text { ium } \\
\text { means }\end{array}$} \\
\hline & Tabat & \begin{tabular}{|c|}
$\begin{array}{c}\text { Wad } \\
\text { ahmed }\end{array}$ \\
\end{tabular} & L4 & L7 & L12 & L14 & L16 & L25 & L32 & L34 & \\
\hline$\cdot$ & $0.22^{\mathrm{ijk}}$ & $0.83^{\text {defghijk }}$ & $0.21^{\mathrm{ijk}}$ & $0.16^{\mathrm{jk}}$ & $0.41^{\text {ghijk }}$ & $0.03^{\mathrm{k}}$ & $0.13^{\mathrm{jk}}$ & $0.81^{\text {defghijk }}$ & $0.73^{\text {defghijk }}$ & $0.88^{\text {defghijk }}$ & $0.44^{\mathrm{c}}$ \\
\hline 2.5 & $0.48^{\text {fghijk }}$ & $0.97^{\text {defghijk }}$ & $0.23^{\mathrm{ijk}}$ & $0.39^{\text {ghijk }}$ & $0.31^{\text {hijk }}$ & $0.07^{\mathrm{k}}$ & $0.11^{\mathrm{jk}}$ & $0.64^{\text {efghijk }}$ & $0.76^{\text {defghijk }}$ & $0.57^{\text {efghijk }}$ & $0.45^{\mathrm{c}}$ \\
\hline 5 & $0.60^{\text {efghijk }}$ & $0.91^{\text {defghijk }}$ & $0.20^{\mathrm{ijk}}$ & $0.27^{\text {hijk }}$ & $0.32^{\mathrm{hijk}}$ & $0.22^{\mathrm{ijk}}$ & $0.18^{\mathrm{ijk}}$ & $0.41^{\text {ghijk }}$ & $0.67^{\text {efghijk }}$ & $0.56^{\text {efghijk }}$ & $0.43^{\mathrm{c}}$ \\
\hline 10 & $0.96^{\text {defghijk }}$ & $1.36^{\text {bcdefg }}$ & $0.39^{\text {ghijk }}$ & $0.40^{\text {ghijk }}$ & $0.62^{\text {efghijk }}$ & $0.22^{\mathrm{ijk}}$ & $0.29^{\mathrm{hijk}}$ & $0.34^{\text {ghijk }}$ & $0.35^{\text {ghijk }}$ & $0.26^{\text {hijk }}$ & $0.52^{\mathrm{bc}}$ \\
\hline 20 & $0.91^{\text {defghijk }}$ & $1.30^{\text {bcdefgh }}$ & $0.70^{\text {efghijk }}$ & $0.52^{\text {efghijk }}$ & $1.53^{\mathrm{abcde}}$ & $0.23^{\mathrm{ijk}}$ & $0.22^{\mathrm{ijk}}$ & $0.35^{\text {ghijk }}$ & $0.26^{\mathrm{hijk}}$ & $0.27^{\mathrm{hijk}}$ & $0.63^{\mathrm{bc}}$ \\
\hline 30 & $1.04^{\text {defghijk }}$ & $1.46^{\text {abcdef }}$ & $0.75^{\text {defghijk }}$ & $0.65^{\text {efghijk }}$ & $1.13^{\text {cdefghij }}$ & $0.28^{\text {hijk }}$ & $0.27^{\text {hijk }}$ & $0.17^{\mathrm{ijk}}$ & $0.28^{\text {hijk }}$ & $0.28^{\text {hijk }}$ & $0.63^{\mathrm{bc}}$ \\
\hline 40 & $1.21^{\text {bcdefghi }}$ & $1.59^{\text {abcde }}$ & $0.73^{\text {defghijk }}$ & $0.87^{\text {defghijk }}$ & $2.20^{\mathrm{ab}}$ & $0.29^{\mathrm{hijk}}$ & $0.47^{\text {fghijk }}$ & $0.20^{\mathrm{ijk}}$ & $0.18^{\mathrm{ijk}}$ & $0.19^{\mathrm{ijk}}$ & $0.79^{\mathrm{ab}}$ \\
\hline 50 & $2.10^{\mathrm{abc}}$ & $1.75^{\mathrm{abcd}}$ & $0.91^{\text {defghijk }}$ & $1.26^{\text {bcdefgh }}$ & $2.40^{\mathrm{a}}$ & $0.36^{\text {ghijk }}$ & $0.58^{\text {efghijk }}$ & $0.15^{\mathrm{jk}}$ & $0.23^{\mathrm{ijk}}$ & $0.15^{\mathrm{jk}}$ & $0.99^{\mathrm{a}}$ \\
\hline $\begin{array}{c}\text { Genotypes } \\
\text { mean }\end{array}$ & $0.94^{\mathrm{a}}$ & $1.27^{\mathrm{a}}$ & $0.52^{\mathrm{bc}}$ & $0.57^{\mathrm{b}}$ & $1.11^{\mathrm{a}}$ & $0.21^{\mathrm{c}}$ & $0.28^{b c}$ & $0.38^{\mathrm{bc}}$ & $0.43^{b c}$ & $0.40^{\mathrm{bc}}$ & \\
\hline
\end{tabular}

Potassium dichromate was used as a source of chromium. The soil used was clay: sand (2:1). Statistical analysis was done using Duncan multiple range test (DMRT). Values with the same superscript letters are not significantly different at $\mathrm{p}<0.05$

Table 5 Effect of chromium on perooxidase activity (u/mg protein) of the ten sorghum genotypes at the $12^{\text {th }}$ day after chromium application at eight different concentrations) to two-week-old seedlings

\begin{tabular}{|c|c|c|c|c|c|c|c|c|c|c|c|}
\hline $\begin{array}{c}\text { Chromium } \\
\text { concentrations } \\
(\mathbf{p p m})\end{array}$ & Tabat & $\begin{array}{c}\text { Wad } \\
\text { ahmed }\end{array}$ & $\mathbf{L 4}$ & $\mathbf{L 7}$ & $\mathbf{L 1 2}$ & $\mathbf{L 1 4}$ & $\mathbf{L 1 6}$ & $\mathbf{L 2 5}$ & $\mathbf{L 3 2}$ & $\mathbf{L 3 4}$ & $\begin{array}{c}\text { Chromium } \\
\text { means }\end{array}$ \\
\hline & $0.11^{\mathrm{a}}$ & $0.09^{\mathrm{a}}$ & $0.09^{\mathrm{a}}$ & $0.34^{\mathrm{a}}$ & $0.11^{\mathrm{a}}$ & $0.09^{\mathrm{a}}$ & $0.13^{\mathrm{a}}$ & $0.12^{\mathrm{a}}$ & $0.21^{\mathrm{a}}$ & $0.14^{\mathrm{a}}$ & 0.14 \\
\hline 0 & $0.12^{\mathrm{a}}$ & $0.11^{\mathrm{a}}$ & $0.09^{\mathrm{a}}$ & $0.20^{\mathrm{a}}$ & $0.13^{\mathrm{a}}$ & $0.07^{\mathrm{a}}$ & $0.17^{\mathrm{a}}$ & $0.15^{\mathrm{a}}$ & $0.14^{\mathrm{a}}$ & $0.20^{\mathrm{a}}$ & 0.14 \\
\hline 2.5 & $0.13^{\mathrm{a}}$ & $0.16^{\mathrm{a}}$ & $0.18^{\mathrm{a}}$ & $0.21^{\mathrm{a}}$ & $0.12^{\mathrm{a}}$ & $0.11^{\mathrm{a}}$ & $0.14^{\mathrm{a}}$ & $0.14^{\mathrm{a}}$ & $0.15^{\mathrm{a}}$ & $0.26^{\mathrm{a}}$ & 0.16 \\
\hline 5 & $0.13^{\mathrm{a}}$ & $0.14^{\mathrm{a}}$ & $0.17^{\mathrm{a}}$ & $0.23^{\mathrm{a}}$ & $0.13^{\mathrm{a}}$ & $0.18^{\mathrm{a}}$ & $0.14^{\mathrm{a}}$ & $0.14^{\mathrm{a}}$ & $0.16^{\mathrm{a}}$ & $0.25^{\mathrm{a}}$ & 0.17 \\
\hline 10 & $0.13^{\mathrm{a}}$ & $0.13^{\mathrm{a}}$ & $0.20^{\mathrm{a}}$ & $0.27^{\mathrm{a}}$ & $0.14^{\mathrm{a}}$ & $0.14^{\mathrm{a}}$ & $0.15^{\mathrm{a}}$ & $0.15^{\mathrm{a}}$ & $0.18^{\mathrm{a}}$ & $0.23^{\mathrm{a}}$ & 0.17 \\
\hline 30 & $0.17^{\mathrm{a}}$ & $0.13^{\mathrm{a}}$ & $0.23^{\mathrm{a}}$ & $0.30^{\mathrm{a}}$ & $0.15^{\mathrm{a}}$ & $0.18^{\mathrm{a}}$ & $0.17^{\mathrm{a}}$ & $0.16^{\mathrm{a}}$ & $0.31^{\mathrm{a}}$ & $0.25^{\mathrm{a}}$ & 0.20 \\
\hline 40 & $0.21^{\mathrm{a}}$ & $0.15^{\mathrm{a}}$ & $0.20^{\mathrm{a}}$ & $0.29^{\mathrm{a}}$ & $0.22^{\mathrm{a}}$ & $0.29^{\mathrm{a}}$ & $0.18^{\mathrm{a}}$ & $0.18^{\mathrm{a}}$ & $0.29^{\mathrm{a}}$ & $0.28^{\mathrm{a}}$ & 0.23 \\
\hline 50 & $0.21^{\mathrm{a}}$ & $0.14^{\mathrm{a}}$ & $0.23^{\mathrm{a}}$ & $0.35^{\mathrm{a}}$ & $0.20^{\mathrm{a}}$ & $0.20 \mathrm{a}$ & $0.32 \mathrm{a}$ & $0.19^{\mathrm{a}}$ & $0.36^{\mathrm{a}}$ & $0.32^{\mathrm{a}}$ & 0.25 \\
\hline $\begin{array}{c}\text { Genotypes } \\
\text { means }\end{array}$ & $0.15^{\mathrm{c}}$ & $0.13^{\mathrm{c}}$ & $0.18^{\mathrm{bc}}$ & $0.27^{\mathrm{a}}$ & $0.15^{\mathrm{c}}$ & $0.16^{\mathrm{c}}$ & $0.17^{\mathrm{bc}}$ & 0.15 & $0.23^{\mathrm{ab}}$ & $0.24^{\mathrm{a}}$ & \\
\hline
\end{tabular}

Potassium dichromate was used as a source of chromium. The soil used was clay: sand (2:1). Statistical analysis was done using Duncan multiple range test (DMRT). Values with the same superscript letters are not significantly different at $\mathrm{p}<0.05$ 
Table 6 Effect of chromium on perooxidase activity (u/mg protein) of the ten sorghum genotypes at the $15^{\text {th }}$ day after chromium application at eight different concentrations) to two-week-old seedlings

\begin{tabular}{|c|c|c|c|c|c|c|c|c|c|c|c|}
\hline \multirow{2}{*}{$\begin{array}{c}\text { Chromium } \\
\text { concentrations } \\
(\mathbf{p p m})\end{array}$} & \multicolumn{10}{|c|}{ Sorghum genotypes } & \multirow[b]{2}{*}{$\begin{array}{c}\text { Chromium } \\
\text { means }\end{array}$} \\
\hline & Tabat & $\begin{array}{c}\text { Wad } \\
\text { ahmed }\end{array}$ & L4 & L7 & L12 & L14 & L16 & $\mathbf{L} 25$ & L32 & L34 & \\
\hline 0 & $0.01^{\mathrm{a}}$ & $0.08^{\mathrm{a}}$ & $0.05^{\mathrm{a}}$ & $0.12^{\mathrm{a}}$ & $0.08^{\mathrm{a}}$ & $0.08^{\mathrm{a}}$ & $0.06^{\mathrm{a}}$ & $0.15^{\mathrm{a}}$ & $0.12^{\mathrm{a}}$ & $0.03^{\mathrm{a}}$ & $0.08^{\mathrm{e}}$ \\
\hline 2.5 & $0.01^{\mathrm{a}}$ & $0.11^{\mathrm{a}}$ & $0.06^{\mathrm{a}}$ & $0.15^{\mathrm{a}}$ & $0.09^{\mathrm{a}}$ & $0.10^{\mathrm{a}}$ & $0.07^{\mathrm{a}}$ & $0.21^{\mathrm{a}}$ & $0.13^{\mathrm{a}}$ & $0.13^{\mathrm{a}}$ & $0.11^{\mathrm{de}}$ \\
\hline 5 & $0.02^{\mathrm{a}}$ & $0.14^{\mathrm{a}}$ & $0.10^{\mathrm{a}}$ & $0.17^{\mathrm{a}}$ & $0.10^{\mathrm{a}}$ & $0.09^{\mathrm{a}}$ & $0.17^{\mathrm{a}}$ & $0.20^{\mathrm{a}}$ & $0.18^{\mathrm{a}}$ & $0.13^{\mathrm{a}}$ & $0.13^{\mathrm{de}}$ \\
\hline 10 & $0.03^{\mathrm{a}}$ & $0.17^{\mathrm{a}}$ & $0.12^{\mathrm{a}}$ & $0.19^{\mathrm{a}}$ & $0.12^{\mathrm{a}}$ & $0.15^{\mathrm{a}}$ & $0.35^{\mathrm{a}}$ & $0.24^{\mathrm{a}}$ & $0.28^{\mathrm{a}}$ & $0.14^{\mathrm{a}}$ & $0.18^{\mathrm{cd}}$ \\
\hline 20 & $0.03^{\mathrm{a}}$ & $0.27^{\mathrm{a}}$ & $0.15^{\mathrm{a}}$ & $0.19^{\mathrm{a}}$ & $0.13^{\mathrm{a}}$ & $0.18^{\mathrm{a}}$ & $0.35^{\mathrm{a}}$ & $0.25^{\mathrm{a}}$ & $0.28^{\mathrm{a}}$ & $0.15^{\mathrm{a}}$ & $0.20^{\mathrm{bc}}$ \\
\hline 30 & $0.04^{\mathrm{a}}$ & $0.29^{a}$ & $0.18^{\mathrm{a}}$ & $0.19^{\mathrm{a}}$ & $0.33^{\mathrm{a}}$ & $0.20^{\mathrm{a}}$ & $0.37^{\mathrm{a}}$ & $0.25^{\mathrm{a}}$ & $0.27^{\mathrm{a}}$ & $0.28^{\mathrm{a}}$ & $0.24^{\mathrm{bc}}$ \\
\hline 40 & $0.08^{\mathrm{a}}$ & $0.31^{\mathrm{a}}$ & $0.23^{\mathrm{a}}$ & $0.19^{\mathrm{a}}$ & $0.34^{\mathrm{a}}$ & $0.25^{\mathrm{a}}$ & $0.38^{\mathrm{a}}$ & $0.31^{\mathrm{a}}$ & $0.28^{\mathrm{a}}$ & $0.32^{\mathrm{a}}$ & $0.27^{\mathrm{b}}$ \\
\hline 50 & $0.18^{\mathrm{a}}$ & $0.41^{\mathrm{a}}$ & $0.23^{\mathrm{a}}$ & $0.26^{\mathrm{a}}$ & $0.53^{\mathrm{a}}$ & $0.24^{\mathrm{a}}$ & $0.42^{\mathrm{a}}$ & $0.48^{\mathrm{a}}$ & $0.42^{\mathrm{a}}$ & $0.37^{\mathrm{a}}$ & $0.35^{\mathrm{a}}$ \\
\hline $\begin{array}{l}\text { Genotypes } \\
\text { means }\end{array}$ & $0.05^{\mathrm{c}}$ & $0.22^{\mathrm{ab}}$ & $0.14^{\mathrm{b}}$ & $0.18^{\mathrm{ab}}$ & $0.21^{\mathrm{ab}}$ & $0.16^{\mathrm{b}}$ & $0.27^{\mathrm{a}}$ & $0.26^{\mathrm{a}}$ & $0.25^{\mathrm{a}}$ & $0.19^{\mathrm{ab}}$ & \\
\hline
\end{tabular}

Potassium dichromate was used as a source of chromium. The soil used was clay: sand (2:1). Statistical analysis was done using Duncan multiple range test (DMRT). Values with the same superscript letters are not significantly different at $\mathrm{p}<0.05$

\section{Discussion}

The eight chromium concentrations used in this study generally induced significant differences in catechol oxidase activities on the 8,12 and 15 days of treatments, and those of peroxidase after $8^{\text {th }}$ and $15^{\text {th }}$ days. Generally, the levels of both enzymes were progressively increased with the increase in chromium concentrations. The $\mathrm{H}_{2} \mathrm{O}_{2}$ levels were increased in both roots and leaves of sorghum treated with either $50 \mu \mathrm{M}$ hexavalent chromium or $100 \mu \mathrm{M}$ trivalent chromium Chatterjee and Chatterjee, (2000) who found that $\mathrm{H}_{2} \mathrm{O}_{2}$ levels was increased in both roots and leaves of sorghum treated with either $50 \mu \mathrm{M}$ hexavalent chromium or $100 \mu \mathrm{M}$ trivalent chromium. The activity of antioxidant enzymes was increased even at low heavy metal concentrations Gwozdz et al. (1997) found that at lower heavy metal concentrations, activity of antioxidant enzymes was increased. The present results suggest that, the levels of activities of catechol oxidase and peroxidase enzymes increased following treatment with different concentrations of hexavalent chromium. This elevation after the $8^{\text {th }}, 12^{\text {th }}$ and $15^{\text {th }}$ day from exposure might be a mechanism of the plant for the enzymes to engage in antioxidant defense. The plant cells have evolved antioxidant defense mechanisms to combat the danger posed by the presence of reactive oxygen species. These include enzymatic mechanisms involving antioxidant enzymes (Meloni et al., 2003). Modification of the plant antioxidant defense system has been reported to enhance tolerance to oxidative stress (Rai et al., 2004; Mishra et al., 2006a, b; Meng et al., 2007; Dazy et al., 2008). Alteration of antioxidant enzymes may be due to the synthesis of new iso enzyme for enhancement of the activity of pre existing enzymes for the metabolism of ROS (Kang et al., 1999). Significant differences were detected among the ten sorghum genotypes in both enzymes, of growth period after chromium treatment application with exception. Generally, with exception of the decreased levels of catechol and peroxidase in L25, L32 and L34 on the $8^{\text {th }}$ day of treatment, the levels of both enzymes in the other genotypes increased with the increase in the chromium concentrations. Similar results were reported by Samantary (2002) who found that, the enzyme activity varied among the chromium tolerant and chromium sensitive ones in mung bean plant. Furthermore Divya (1999) reported a decrease in the activity of ascorbate peroxidase with increase in cadmium concentration in radical and plumule of pea seedling and decrease was prominent in susceptible than the tolerant variety. The decline in activity of this oxidative and antioxidant enzyme has been ascribed to inhibition of enzyme biosynthesis and the denaturation of enzyme proteins (Mohapatra, 1995).

\section{Conclusion}

The present results indicate a fluctuation in the activity of both catechol oxidase and peroxidase. Such fluctuation seemed to depend on genotypes and/or the elapsed time following treatment with $\mathrm{Cr}$. Therefore, the differential changes in these antioxidant enzymes might point at a differential degree of stress induced in sorghum genotypes by $\mathrm{Cr}$, So, the changes in activity of catechol oxidase and peroxidase might be in relation with plant tolerance to $\mathrm{Cr}$. Hence, the variation in the 
enzymatic activity might be used as markers for oxidative stress and tolerance of sorghum to $\mathrm{Cr}$ phytotoxicity, the point that needs further investigation.

\section{References}

Ali NA, Ater M, SunaharaGl, Robidoux PY (2004) Phytotoxicity and bioacuumulation of copper and chromiumusing barley (HordeumVulgare L.) in spiked artificial and natural forestsoils. Ecotoxicology and environmental safety. 57: 363374

Babel S, Opiso EM (2007) Removal of Cr from synthetic wastewater by sorption into volcanic ash soil.Int. J. Environ. Sci. Tech., 4 (1), 99-107

Bradford MM (1976) A rapid and sensitive method for quantitation of microgram quantities of protein utilization the principle of protein- dye binding.Analytical Biochemistry.2:248 - 254.

Chatterjee J,Chatterjee C (2000) Phytotoxicity of cobalt, chromium and copper in cauliflower. Environmental Pollution 109: 69-74.

Dazy M, Beraud E, Cotelle S, Meux E, Masfaraud JF,Ferard JF (2008)Antioxidant enzyme activities as affected by trivalent and hexavalent chromium species

FontinalisantipyreticaHedw.Chemosphere, $\quad 73$ : 281-290

Divya D (1999) Screening of pea cultivars for cadmium toxicity and mechanism of cadmium-calcium interaction. Ph.D. Thesis, India: C.C.S. Harayana Agricultural University, Hisar.

Du Y, He JH, Chen JJ, Wei XG, Yang XQ, WangSY, $\mathrm{He} W B(2003)$ Effects of heavy metals of Pb,Cd and $\mathrm{Cr}$ on the growth of vegetables and their uptake.Acta HorticulturaeSinica, 30 (1), 51-55

Dube B, Tewari K, Chatterjee J, Chatterjee C (2003) Excess chromium alters uptake and translocation of certain nutrients in citrullus. Chemosphere 53: 1147-1153.

Gomez, K.A. and Gomez, A. A. (1984) Statistical Procedures for Agricultural Research.John-Willey and Sons, New York.

Gwozdz EA, Przymusinski R, Rucinska R, Deckert J (1997) Plant cell responses to heavy metals: molecular and physiological aspects. Acta Physiol Plant. 19:459 - 465.

Huffman EWD, Allaway WH (1973) Growth of plants in solution culture containing low levels of chromium. Plant Physiol., 52: 72-75.

Jain R, Srivastava S, Madan VK, Jain R (2000) Influence of chromium on growth and cell division of sugarcane. Indian J. Plant Physi., 5 (3), 228-231

Kang HG, Jun SH, Kim J, Kawaide H, Kamiya Y, An G (1999) Cloning and molecular analyses of a gibberellin 20-oxidase gene expressed specifically in developing seeds of watermelon. Plant Physiol.121: 373-382.

Liu DH, Jiang WS, Li MX (1992) Effects of trivalent and hexavalent chromium on root growth and cell division of Allium cepa. Hereditas, 117: 23-29.

MandelmanD, Jamal J, Poulos TL (1998) Identification of two electron transfer sites in ascorbate peroxidase using chemical modification, enzyme kinetics and crystallography.Biochemistry, 73: 17610 - 17617.

Meloni DA, Oliva MA, Martinez CA, Cambrai AJ (2003) Photosynthesis and activity of superoxide dismutase, peroxidase and glutathione reductase in cotton under salt stress. Environmental and Experimental Botany. 49: 69-76.

Meng QM, Zouj Z, Jinng WS, Liu DH (2007) Effect of $\mathrm{Cu} 2+$ concentration on growth, antioxidant enzyme activity and malondialdehyde content in garlic (Allium sativum L.). Acta BiologicaCracoviensia Series Botanica .49: 95-101.

Mertz, W. 1967.Biological role of chromium.Fed. Proc., 26: 186-193

Mishra S, Srivastava S, Tripathi RD, Govindarajan R, Kuriakose SV, Prasad MNV (2006a) Phytochelatin synthesis and response of antioxidants during cadmium stress inBacopamonnieriL. Plant Physiol. Biochem. 44, 25-37.

Mishra S, Srivastava S, Tripathi RD, Kumar R, Seth CS, Gupta DK (2006b) Lead detoxification by coontail (Ceratophyllumdemersum L.) involves induction of phytochelatins and antioxidant system in response to its accumulation. Chemosphere, 65, 1027-1039.

Mohapatra S (1995) Chromium toxicity and water stress interaction in green gram Vigna radiate L. during seed germination and seedling growth. M.Phil. Thesis, India: Utkal University. Bhubaneshwar.

Nath K, Saini S, Sharma YK (2005) Chromium in tannery industry effluent and its effect on plant metabolism and growth. J. Environ. Biol., 26 (2), 197- 204

Parmar NG, Vithalani SD, Chanda SV (2002) Alteration in growth and peroxidase activity by heavy metals in Phaseolusseedlings.Acta Physiolog.Plant. 24 (1), 89-95

Rai V, Vajpayee P, Mehratra S (2004) Effects of Chromium accumulation on photo-synthetic pigments, oxidative stress defense system, nitrate reduction, proline level and eugenol content of Ocimumtenuiflorum L., Plant Sci. 167: 1159-1169.

Samantary S (2002) Biochemical responses of Crtolerant and $\mathrm{Cr}$-sensitive mung bean cultivars grown on varying levels of chromium. Chemosphere. 47: 1065-1072 
Scoccianti V, Crinelli R, TirilliniB,Mancinelli V, Speranza A (2006) Uptake and toxicity of Cr (III) in celery seedlings. Chemosphere. 64 (10), 1695 1703

Sharma DC, Pant RC (1994) Chromium uptake its effectson certain plant nutrients in Maize (Zea mays L. cv. Ganga 5). Journal of Environmental Science andHealth, Part A 29: 941-948

Sharma DC, Chatterjee C, Sharma CP (1995) Chromium accumulation by barley seedlings (Hordeumvulgare L.). J. Exp. Bot. 25: 241-251

Srivastava S,Prakash S, Srivastava MM (1999) Chromium mobilization and plant availability - the impact of organic complexing agents. Plant Soil, 212: 203-208.

Tremoliere M, Bieth JB (1984) Determination of catechol oxidase activity using spectroscopic methods. Phyochemistry. 23: $501-505$.

Venkateswaran P, Vellaichamy S, Palanivelu, K (2007) Speciation of heavy metals in electroplating industry sludge and wastewater residue using inductively coupled plasma. Int. J. Environ. Sci. Tech., 4 (4), 497-504

Wong JWC, Lai KM, Su DS, Fang M (2001) Availability of heavy metals for Brassica chinensisgrown in an acidic loamy soil amended with a domestic and an industrial sewage sludge. Water Air Soil Poll. 128 (3-4), 339-353

Zayed A, Lytle CM,Qian JH, Terry N (1998) Chromium accumulation, translocation and chemical speciation in vegetable crops, planta. 206: 293-299.

Zayed AM, Terry N (2003) Chromium in the environment: factors affecting biological remediation. Plant Soil, 249: 139-156.

Zhen Z Q, FengWH, Bian SP, Zheng JM, Zhang LZ, Xing SL (1987) Study on fate of pollutant chromium in the Agro-Ecosystem. J. Environ. Sci., 8: 14-19.

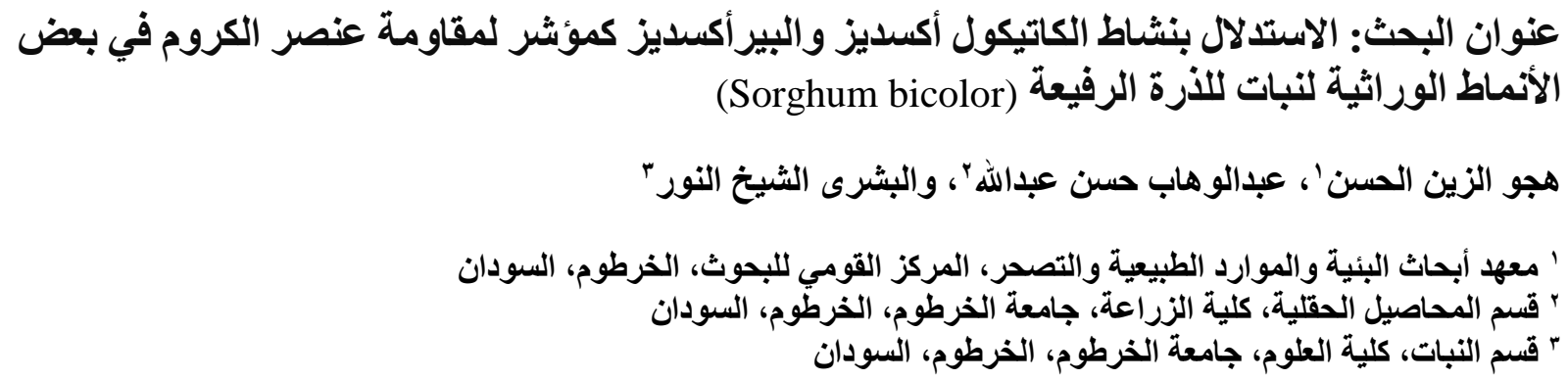

هدفت الاراسة الحالية الىى تقيميم أثر عنصر الكروم على نشاط انزيمي الكاتيكول أكسديز والبيز أكسديز

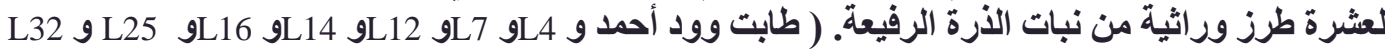

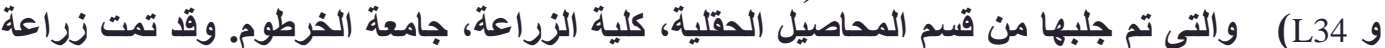

البذور في اكياس بولثيرين يحتوى كل منها على Y كجم من ثربة خليط من الطين والرمل (Y : l). وبعد

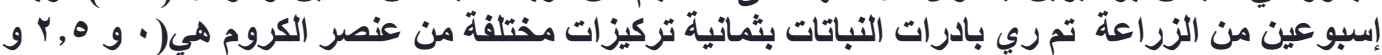

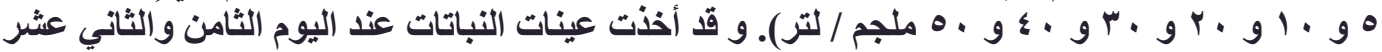

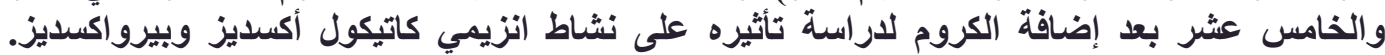

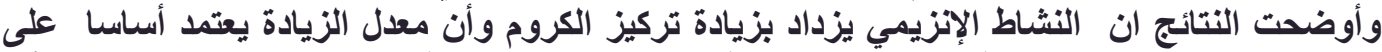

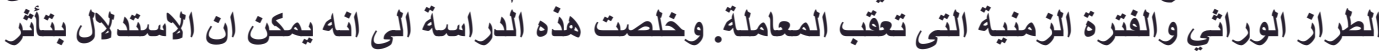

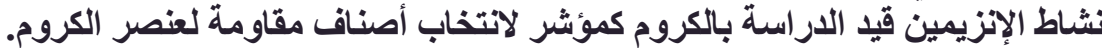

